\title{
Measurement and modeling of diffusion time dependence of apparent diffusion coefficient and fractional anisotropy in prostate tissue ex vivo
}

Roger Bourne *, Sisi Liang, Eleftheria Panagiotaki, Andre Bongers, Paul Sved, and Geoffrey Watson

\section{* Corresponding author:}

Dr Roger Bourne

Discipline of Medical Radiation Sciences | Faculty of Health Sciences

THE UNIVERSITY OF SYDNEY

75 East Street | Lidcombe | NSW | 2141

T +612 86275289 (Lifehouse, Camperdown) | +6129036 7350 (Cumberland)

F +61293519146

E roger.bourne@sydney.edu.au

Word count: 3186

Sponsors: Australian National Health and Medical Research Council grant 1026467.

UK-EPSRC grant EP/N021967/1

Keywords: diffusion, ADC, FA, anisotropy, restriction, modeling, prostate

Abbreviations: ADC: apparent diffusion coefficient; FA: fractional anisotropy; DTI: diffusion tensor imaging; MD: mean diffusivity 


\section{Abstract}

The purpose of this study was to measure and model the diffusion time dependence of apparent diffusion coefficient (ADC) and fractional anisotropy (FA) derived from conventional prostate DWI methods as used in recommended mpMRI protocols. Diffusion tensor imaging was performed at 9.4 Tesla with three radical prostatectomy specimens, with diffusion times in the range $10-120 \mathrm{~ms}$ and $b$-values $0-3000 \mathrm{~s} / \mathrm{mm}^{2}$. ADC and FA were calculated from DTI measurements at $b$-values of 800 and $1600 \mathrm{~s} / \mathrm{mm}^{2}$. Independently, a two component model (restricted isotropic plus Gaussian anisotropic) was used to synthesize DTI data from which ADC and FA were predicted and compared with the measured values. Measured ADC and FA exhibited a diffusion time dependence which was closely predicted by the two component model. ADC decreased by $\sim 0.10-0.15 \mu \mathrm{m}^{2} / \mathrm{ms}$ as diffusion time increased from 10 to $120 \mathrm{~ms}$. FA increased with diffusion time at $b$-values of 800 and $1600 \mathrm{~s} / \mathrm{mm}^{2}$ but was predicted to be independent of diffusion time at $b=3000 \mathrm{~s} / \mathrm{mm}^{2}$. Both ADC and FA exhibited diffusion time dependence which could be modeled as two unmixed water pools - one having isotropic restricted dynamics, and the other unrestricted anisotropic dynamics. These results highlight the importance of considering and reporting diffusion times in conventional ADC and FA calculations and protocol recommendations, and inform the development of improved diffusion methods for prostate cancer imaging. 


\section{Introduction}

Diffusion-weighted imaging (DWI) is an important component of the multiparametric MRI (mpMRI) scan for prostate cancer $(1,2)$. DWI provides a more reliable estimate of cancer grade and volume than T2 and DCE (dynamic contrast enhancement) (3-5), and an apparent diffusion coefficient (ADC) derived from the DWI signal attenuation correlates moderately with cancer Gleason grade $(6,7)$.

\section{Apparent diffusion coefficient (ADC)}

DWI contrast variations reflect intra-voxel water mobility. The water displacement sensitivity of the DWI scan method is typically described by the ' $b$-value', a term that combines the diffusion encoding gradient strength and the effective diffusion time. For a conventional pulsed gradient DWI method having two gradient lobes of strength $G$, lobe duration $\delta$, and lobe separation $\Delta-\delta$, the $b$-value is defined as:

$$
b=\gamma^{2} G^{2} \delta^{2}(\Delta-\delta / 3)
$$

where $\gamma$ is the ${ }^{1} \mathrm{H}$ magnetogyric ratio (8). Clearly, any specific $b$-value can be generated by an infinite number of combinations of $G, \delta$, and $\Delta$. In practice the implemented values vary from scanner to scanner and may depend on available gradient strength $\left(G_{\max }\right)$, the range of $b$-values selected, and the manufacturer's software. When $G_{\max }$ is limited, high $b$-values are achieved by increasing $\Delta$. The effective diffusion time, $\Delta-\delta / 3$, is an important parameter as it determines the spatial scale of the microstructural interactions affecting the average water displacement. Unfortunately, most clinical scanner software does not display the implemented $\delta$ and $\Delta$ values which are automatically selected by the software, and they are rarely reported in clinical DWI studies. Diffusion time considerations have also been neglected in the mpMRI method consensus statements $(1,2)$.

In the majority of clinical studies ADC is derived from a monoexponential model of the DWI signal attenuation: 


$$
A D C=-\ln \left(S / S_{0}\right) / b
$$

where $S$ is the signal at diffusion weighting $b$, and $S_{0}$ the signal in the absence of diffusion weighting $(b=0)$. A major limitation of this method is that the monoexponential model assumes a Gaussian water displacement probability that is now well-known to be invalid in prostate tissue (9). The diffusion properties of prostate tissue are microscopically heterogeneous $(10,11)$, and demonstrate a diffusion-time dependence that can be modeled as a restricted diffusion component both in vivo (12) and ex vivo (13). The presence of a significant restricted pool of spins would be expected to lead to a diffusion time-dependence of calculated ADC, however, although this result is implicit in some multicomponent models of the prostate DWI signals (eg. $(12,13))$, such dependence has not been specifically investigated.

\section{Fractional anisotropy $(F A)$}

Diffusion tensor imaging (DTI) extends the monoexponential ADC model to account for diffusion anisotropy, but does not account for diffusion restriction. A summary parameter, fractional anisotropy (FA), describes the degree of diffusion anisotropy but has not yet been demonstrated to have any clinical value in prostate disease assessment. Despite high microscopic anisotropy in the fibromuscular stroma (11), ex vivo whole organ studies show generally low anisotropy at clinical voxel volumes and wide FA differences between prostates (14). It has been argued that the relatively low signal-to-noise ratio (SNR) of clinical DWI measurements leads to artifactually high FA estimates $(15,16)$.

\section{Aims}

Notwithstanding the significant current clinical value of DWI and ADC for assessment of prostate cancer, it is possible that unreported and unrecognized diffusion time differences between published studies have contributed to inconsistencies in results for both ADC and FA. A recent clinical study reported that both ADC and FA showed diffusion time dependence (17). The aim of the study reported here was to directly assess the effects of diffusion time and noise on ADC and FA measurements in radical prostatectomy specimens. A secondary aim was to model the diffusion time effects as a simple combination of an isotropic restricted component and an anisotropic unrestricted component. 


\section{Methods}

Three radical prostatectomy specimens were collected immediately after surgery with institutional ethics approval and written informed consent from patients (Specimen 1: $50 \mathrm{~g}, 62$ yo, PSA 8.3 ng/mL. Specimen 2: 44 g, 62 yo, PSA $5.0 \mathrm{ng} / \mathrm{mL}$. Specimen 3:, 89 g, PSA 6.5 $\mathrm{ng} / \mathrm{mL}, 60$ yo). Specimens were fixed overnight in $10 \%$ neutral buffered formalin and then immersed in $1 \mathrm{~L} 0.5 \%(\mathrm{w} / \mathrm{v})$ saline overnight to remove formalin prior to imaging (14). The prostate was imaged suspended on a 5-mm saline-filled NMR tube inserted through the urethra and mounted in brackets in a plastic casing that maintained the tube axis parallel to and approximately $5 \mathrm{~mm}$ above the magnet $\mathrm{z}$-axis. The prostate was wrapped in parafilm to minimize dehydration during imaging. After imaging the prostate was returned to the pathology department for routine processing. The distribution of cancer is illustrated for a single central slice of each prostate in Fig. 3, however, it should be noted that due to the small number of samples and imprecise alignment of DWI and histology slices no attempt was made to correlate results with prostate pathology.

Imaging was performed at room temperature $\left(22^{\circ} \mathrm{C}\right)$ on a $9.4 \mathrm{~T}$ Bruker (Karlsruhe, Germany) BioSpec Avance III 94/20 system equipped with a 72-mm internal diameter quadrature radiofrequency coil and BGA-12S HP gradients with maximum strength $660 \mathrm{mT} / \mathrm{m}$ and slew rate $4570 \mathrm{~T} / \mathrm{m} / \mathrm{s}$. Imaging was performed transaxial to the urethra with the imaging planes oriented orthogonal to the 5-mm NMR tube with 2-mm slice thickness and a $2 \mathrm{~mm}$ gap between slices. FOV $=60 \times 60 \mathrm{~mm}$. Matrix $=64 \times 64$. DWI measurements were performed using a PGSE sequence preceded by the acquisition of two reference ' $b=0$ ' images. To investigate the effect of diffusion time on ADC and FA, DTI acquisitions used a six-direction scheme with nominal $b$ values 800 and $1600 \mathrm{~s} / \mathrm{mm}^{2}$ and a wide range of diffusion times $(10-120 \mathrm{~ms})$. Three-direction DWI data acquired with eight $b$-values $\left(100-3000 \mathrm{~s} / \mathrm{mm}^{2}\right)$ and four diffusion times $(\Delta=10,20$, $40,80 \mathrm{~ms}$ ) were combined with the $\Delta=20 \mathrm{~ms}$ DTI measurements to fit a two component 'Zeppelin-sphere' model (13).

All measurements employed the minimum available TE $(\sim \Delta+8 \mathrm{~ms})$ to maximize signal-to-noise ratio (SNR). DWI parameters including SNR estimates are detailed in Tables 1 and 2. There were small differences in acquisition parameters between the three prostates according to 
available scan time. SNR $=S / N$ of the measurements was estimated for each diffusion time in a large region of interest manually drawn inside single mid-organ slice of each prostate. The noise level $(N)$ was defined as the standard deviation of the difference between the ROI voxel values in the two ' $b=0$ ' reference images. The signal $(S)$ was defined as the mean ROI voxel value taken over all three or six gradient orientations.

\section{Model fitting. Calculation of ADC and FA.}

Intraprostatic voxels were selected for analysis by manual definition of multi-slice masks that excluded the capsule, periprostatic fat, and the urethra and NMR tube. Masking resulted in the selection of 3957, 3510, and 4680 voxels from Prostates 1, 2, and 3 respectively.

An overview of the signal analysis and modeling strategy is provided in Fig. 1. DTI and Zeppelin-sphere (18) models were fitted to the individual voxel data using the LevenbergMarquardt minimization algorithm in the open source Camino toolkit (19). To minimize any possible T2 effects resulting from the variable echo times, data acquired for each diffusion time were normalized to the ' $b=0$ ' reference images for that diffusion time.

In this study we treat the mean diffusivity of the calculated DTI tensor as the ADC that would be obtained from a conventional 3-direction clinical DWI scan. The typically very low FA observed in prostate tissue at the voxel volumes used in this study indicate there would be very little orientation dependence of the ADC derived from a 3-direction DWI measurement (14). To estimate the effect of an isotropic restricted signal component on the ADC and FA at each measured diffusion time the Zeppelin-sphere model was fitted to the combined 3-direction data (Table 2) and $\Delta=20 \mathrm{~ms} 6$-direction data for each voxel. We used only the $\Delta=20 \mathrm{~ms}$ DTI data to define the zeppelin parameters while maximizing the independence the measured and predicted ADC and FA data. The Zeppelin-sphere model has previously been demonstrated to have a consistently maximal information content for a multi- $b$, multi- $\Delta$ measurements in fixed prostate tissue when compared to isotropic models and models that do not account for diffusion restriction (13)). The model defines two water pools representing isotropic restricted diffusion in an impermeable spherical pore (the 'sphere'), and unrestricted anisotropic Gaussian diffusion described by a rotationally symmetric tensor (the 'zeppelin'). There is assumed to be no significant water exchange between the two pools during the measurement. Note that the closely 
related but more highly parameterized 'tensor-sphere' model does not have any consistent information content superiority for prostate tissue (13). The Zeppelin-sphere model has five free parameters which were fitted with constraints as described in Table 3.

The fitted Zeppelin-sphere model parameters were used to synthesize zero-noise 6-direction DTI data at $b=800,1600$, and $3000 \mathrm{~s} / \mathrm{mm}^{2}$ for each diffusion time. The DTI model was then used to fit the synthetic data and calculate ADC (tensor mean diffusivity) and FA. We include the $b=$ $3000 \mathrm{~s} / \mathrm{mm}^{2}$ prediction in light of the recent strong interest in high $b$-value DWI.

The quality of the fit of the Zeppelin sphere model to a representative sample of voxels from each prostate is illustrated in Supplementary Fig. S1.

\section{Results}

Fig. 2 shows variation of average measured ADC and FA at $b=800$ and $1600 \mathrm{~s} / \mathrm{mm}^{2}$, and the average predicted ADC and FA at $b=800,1600$, and $3000 \mathrm{~s} / \mathrm{mm}^{2}$. For reference, we also show $b=1600 \mathrm{~s} / \mathrm{mm}^{2} \mathrm{FA}$ data previously reported from measurements at larger voxel size (14). In all three prostates $\mathrm{ADC}$ and FA showed similar diffusion time dependence over the range $\Delta=10-$ $80 \mathrm{~ms}$. Above $\Delta=80 \mathrm{~ms}$ measured ADC and FA increased markedly in the noisier data from Prostates 1 and 2. At $b=3000 \mathrm{~s} / \mathrm{mm}^{2}$ the Zeppelin-sphere model predicts that average FA would be very low $(<0.2)$ and essentially independent of diffusion time. In contrast the model predicts a stronger diffusion time dependence of ADC at $b=3000$ than at $b=800$ and $1600 \mathrm{~s} / \mathrm{mm}^{2}$.

Fig. 3 illustrates the typical variation in Zeppelin-sphere model parameters within the prostate. In all three prostates the anisotropic zeppelin component had a greater signal fraction and a higher diffusivity than the restricted sphere component.

Fig. 4 shows the voxel-wise correlation between measured and predicted ADC and FA presented as $2 \mathrm{D}$ contour histograms based on voxel count. There was generally a close agreement between measured and predicted ADC at diffusion times up to $\Delta=80 \mathrm{~ms}$. Measured and predicted FA showed a wider variance than ADC, with the measured FA generally being higher than predicted FA in the lower SNR long diffusion time data. 
To illustrate the effect on apparent FA of the isotropic sphere 'background' signal Fig. 5 shows the relationship between DTI-measured FA and the FA of the fitted zeppelin component. As expected the zeppelin FA is generally higher than DTI-based FA, except in the relatively low SNR long diffusion time data from Prostates 1 and 2 where the high noise level generates an artificially high FA in the measurement data.

\section{Discussion}

Several previous studies of water diffusion dynamics in prostate tissue indicate the presence of two main spin pools having distinct diffusivities $(13,20-23)$. Internally, each pool is heterogeneous and shows diffusion time dependence. In vivo prostate DWI studies also point to the presence of two main water pools distinct from the vasculature, one of which can be modeled as a restricted spherical pore $(12,24)$. All of these results suggest there will be a diffusion time dependence of $\mathrm{ADC}$, and possibly also of FA, as measured by a conventional monoexponential model. Indeed, such results have very recently been described for prostate DWI in vivo over a diffusion time range of 21-350 ms (17). In this study we have measured this diffusion time dependence ex vivo and shown that it can be modeled as an isotropic restricted water pool combined with an unrestricted anisotropic pool.

The range of parameter values we observed for the Zeppelin-sphere model are biophysically plausible and in good agreement with a previous multi-component modeling study of four fixed prostate specimens (13), and also the 3-component VERDICT model of in vivo prostate DWI signals (12). The VERDICT model does not include an anisotropic signal component, but its 'extracellular extravascular' and 'sphere' components clearly correspond to the zeppelin and sphere components of the model used here. Our average sphere radius is smaller than the VERDICT sphere, which is probably a result of formalin fixation which is known to cause tissue shrinkage due to protein cross-linking (25).

\section{Effect of diffusion time on ADC}


In all three prostates measured and predicted ADC decreased as diffusion time increased from $\Delta=10$ to $70 \mathrm{~ms}$. This result is consistent with in vivo observations at $b=500 \mathrm{~s} / \mathrm{mm}^{2}$ (17). In Prostates 1 and 2 measured ADC increased above $\Delta \sim 70 \mathrm{~ms}$, especially at $b=800 \mathrm{~s} / \mathrm{mm}^{2}$. The absence of a measured ADC increase above $\Delta \sim 70 \mathrm{~ms}$ in the relatively high SNR Prostate 3 data, and the absence of an increase in the predicted ADC for Prostates 1 and 2 suggest the increase in measured ADC above $\Delta \sim 70 \mathrm{~ms}$ is a noise artifact. The observation of lower ADC at $b=1600$ than at $800 \mathrm{~s} / \mathrm{mm}^{2}$ is likewise consistent with the presence of "slow" and "fast" diffusing water pools - at higher $b$-values the "slow" pool makes a greater contribution to the measured signal.

The absolute ADC change with diffusion time was similar for all three prostates and was very similar at $b=800,1600$, and $3000 \mathrm{~s} / \mathrm{mm}^{2}$. In the typical range of diffusion times employed in clinical imaging ( $40-80 \mathrm{~ms})$ ADC decreased $\sim 0.05 \mu \mathrm{m}^{2} / \mathrm{ms}$. While this change is smaller than the reported average ADC differences between cancer and normal tissue, it is similar to the differences between ADC of adjacent Gleason grades $(6,7)$ and could contribute to an underestimate of the sensitivity and specificity of DWI for cancer detection and grading.

In this study we treated $\mathrm{ADC}$ as equivalent to the mean diffusivity (MD) of a diffusion tensor calculated from a 6-direction DTI measurement. In conventional prostate DWI it is more common to calculate ADC from a 3-direction measurement. If the voxel anisotropy is high then there may be discrepancies between 3-direction $\mathrm{ADC}$ and 6-direction MD according to the orientation of the 3-direction gradients relative to the voxel primary eigenvector. In this study average FA was very low, as was the case in a previous study of seven prostates ex vivo (14), so we believe there are unlikely to be significant differences between our MD-based ADC results and what would be expected from a 3-direction measurement.

\section{Effect of diffusion time on FA}

In all three prostates measured and predicted average FA was low and increased as diffusion time increased at $b=800$ and $1600 \mathrm{~s} / \mathrm{mm}^{2}$, with a smaller increase at the higher $b$-value. Again, this results is consistent with in vivo measurements performed at $b=500 \mathrm{~s} / \mathrm{mm}^{2}$ (17). The low average FA is consistent with earlier reports, including the marked voxel volume dependence of FA (14). The $b=1600 \mathrm{~s} / \mathrm{mm}^{2}$ FA data cannot be directly compared with the previously reported 
data from measurements at larger voxel size $(14,17)$ but the current and earlier data together suggest that voxel size may also affect the diffusion time dependence.

The Zeppelin-sphere model prediction suggested FA to be essentially independent of diffusion time at $b=3000 \mathrm{~s} / \mathrm{mm}^{2}$. There was a very large increase in measured FA at long diffusion times $(\Delta>70 \mathrm{~ms}$ ) in Prostates 1 and 2, but not in Prostate 3. This FA increase mimics (with exaggeration) the measured ADC increase at long diffusion time and low SNR and is also most likely a noise artifact. In contrast to ADC (or MD), the calculation of FA does not average the signals acquired from multiple diffusion directions and is thus more strongly affected by noise (15).

Average FA decreased as $b$-value increased - consistent with the anisotropic signal arising from a high diffusivity water pool (the zeppelin component of the zeppelin-sphere model). At high $b$ values the high diffusivity water signal would be strongly attenuated and the measured signal would be dominated by the isotropic (sphere) component. If we make the tentative hypothesis that the anisotropic high diffusivity signal is predominantly due to extracellular water in the stroma (the stroma makes up the largest volume fraction in normal prostate tissue (26)) then the increase of FA with diffusion time at low $b$-values (where the high diffusivity anisotropic signal dominates) can be interpreted as increasing lateral diffusion hindrance in the roughly parallel packing of stromal myocytes (11).

\section{Limitations}

The results of this study cannot be directly translated to clinical prostate imaging. We report results for just three prostates studied ex vivo. Possibly significant differences from in vivo clinical ADC and FA measurements include tissue perfusion, temperature, fixation, and available range of diffusion times. We used the minimum available TE to maximize the SNR of the measurements. It is possible that some of the variation in ADC and FA we observed could be due to the presence of multiple T2 components, however, variable TE and the dependence of TE on selected $b$-values is a confound that applies equally to conventional in vivo DWI methods. Possible T2 effects on single and multi-component DWI models are an important subject of further investigation. 
The demonstrated diffusion time dependence of ADC and FA in these relatively simple and controlled tissue samples indicates the importance of considering diffusion time in clinical studies (17), and incorporating these effects in advanced DWI techniques.

\section{Clinical significance}

As noted in the Introduction, the possible dependence of reported ADC and FA values on diffusion time has not been widely discussed - particularly in relation to prostate DWI. Our results in this study suggest that both ADC and FA measurements will show some diffusion time dependence, as has recently been observed (17). Some of this time dependence can be attributed to the presence of a significant pool of water exhibiting isotropic restricted diffusion dynamics. In vivo, the success of the isotropic 3-component VERDICT model, which includes a restricted sphere component, also points to a likely diffusion time dependence of ADC measured in vivo. It is possible that unrecognized and unreported differences in diffusion time contribute to differences in reported ADC ranges for both cancer and normal tissue. Furthermore, the ability to discriminate cancer and normal tissue may be diffusion time dependent (17).

Although DWI and ADC are the mainstay of the widely used multiparametric MRI assessment of prostate pathology, further development of the sensitivity and specificity of mpMRI-DWI is desirable and feasible -- especially given the biophysical oversimplification inherent in the monoexponential ADC model.

\section{Conclusions}

Both ADC and FA exhibit diffusion time dependence in fixed prostate tissue. This diffusion time dependence can be modeled as two unmixed water pools - one having isotropic restricted dynamics and the other unrestricted anisotropic dynamics. These results highlight the importance

of considering and reporting diffusion times in conventional ADC and FA calculations and protocol recommendations. 


\section{References}

1. Dickinson L, Ahmed HU, Allen C, Barentsz JO, Carey B, Futterer JJ, Heijmink SW, Hoskin PJ, Kirkham A, Padhani AR, Persad R, Puech P, Punwani S, Sohaib AS, Tombal B, Villers A, van der Meulen J, Emberton M. Magnetic Resonance Imaging for the Detection, Localisation, and Characterisation of Prostate Cancer: Recommendations from a European Consensus Meeting. Eur Urol 2011;59(4):477-494.

2. Padhani AR, Collins D, Hammoud DA, Rustin GJS, Taouli B, Choyke PL, Liu G, Koh DM, Chenevert TL, Thoeny HC, Takahara T, Dzik-Jurasz A, Ross BD, Van Cauteren M. Diffusion-weighted magnetic resonance imaging as a cancer biomarker: consensus and recommendations. Neoplasia 2009;11(2):102-125.

3. Isebaert S, Van den Bergh L, Haustermans K, Joniau S, Lerut E, De Wever L, De Keyzer F, Budiharto T, Slagmolen P, Van Poppel H, Oyen R. Multiparametric MRI for prostate cancer localization in correlation to whole-mount histopathology. Journal of Magnetic Resonance Imaging 2012;37:1392-1401.

4. Selnaes K, Heerschap A, Jensen L, al e. Peripheral Zone Prostate Cancer Localization by Multiparametric Magnetic Resonance at 3 T: Unbiased Cancer Identification by Matching to Histopathology. Investigative Radiology 2012;47(11):624-633.

5. Metzger GJ, Kalavagunta C, Spilseth B, Bolan PJ, Li X, Hutter D, Nam JW, Johnson AD, Henriksen JC, Moench L. Detection of Prostate Cancer: Quantitative Multiparametric MR Imaging Models Developed Using Registered Correlative Histopathology. Radiology 2016;279(3):805-816.

6. Hambrock T, Somford DM, Huisman HJ, van Oort IM, Witjes JA, Hulsbergen-van de Kaa CA, Scheenen T, Barentsz JO. Relationship between apparent diffusion coefficients at 3.0-T MR imaging and Gleason grade in peripheral zone prostate cancer. Radiology 2011;259(2):453-461.

7. Oto A, Yang C, Kayhan A, Tretiakova M, Antic T, Schmid-Tannwald C, Eggener S, Karczmar GS, Stadler WM. Diffusion-weighted and dynamic contrast-enhanced MRI of prostate cancer: correlation of quantitative MR parameters with Gleason score and tumor angiogenesis. American Journal of Roentgenology 2011;197(6):1382-1390.

8. Jones DK, editor. Diffusion MRI. New York: Oxford University Press; 2011.

9. Bourne R, Panagiotaki E. Limitations and Prospects for Diffusion-Weighted MRI of the Prostate. Diagnostics 2016;6(2):21.

10. Bourne RM, Kurniawan N, Cowin G, Stait-Gardner T, Sved P, Watson G, Price WS. Microscopic diffusivity compartmentation in formalin-fixed prostate tissue. Magnetic Resonance in Medicine 2012;68(2):614-620.

11. Bourne R, Kurniawan N, Cowin G, Sved P, Watson G. Microscopic Diffusion Anisotropy in Formalin Fixed Prostate Tissue: Preliminary Findings. Magnetic Resonance in Medicine 2012;68:1943-1948.

12. Panagiotaki E, Chan R, Dikaios H, Ahmed HU, O'Callaghan J, Freeman A, Atkinson D, Punwani S, Hawkes D, Alexander D. Microstructural Characterization of Normal and Malignant Human Prostate Tissue With Vascular, Extracellular, and Restricted Diffusion for Cytometry in Tumours Magnetic Resonance Imaging. Investigative Radiology 2015;50:218-227. 
13. Liang S, Panagiotaki E, Bongers A, Shi P, Sved P, Watson G, Bourne R. Information based ranking of ten compartment models of diffusion weighted signal attenuation in fixed prostate tissue. NMR in Biomedicine 2016;29(5):660-671.

14. Bourne RM, Bongers A, Chatterjee A, Sved P, Watson G. Diffusion anisotropy in fresh and fixed prostate tissue ex vivo. Magnetic Resonance in Medicine 2016;76:626-634.

15. Uribe CF, Jones EC, Chang SD, Goldenberg SL, Reinsberg SA, Kozlowski P. In vivo 3T and ex vivo 7T Diffusion Tensor Imaging of prostate cancer: correlation with histology. Magnetic Resonance Imaging 2015;33(5).

16. Reinsberg S, Brewster J, Payne G, Leach M, deSouza N. Anisotropic diffusion in prostate cancer: fact or artifact? ; 2005; Miami Beach, Florida, USA,. p 269.

17. Lemberskiy G, Rosenkrantz AB, Veraart J, Taneja SS, Novikov DS, Fieremans E. TimeDependent Diffusion in Prostate Cancer. Investigative radiology 2017:(Early view doi: 10.1097/RLI.0000000000000356).

18. Panagiotaki E, Schneider T, Siow B, Hall MG, Lythgoe MF, Alexander DC. Compartment models of the diffusion MR signal in brain white matter: A taxonomy and comparison. Neuroimage 2012;59(3):2241-2254.

19. Cook PA, Bai S, Nedjati-Gilani S, Seunarine KK, Hall MG, Parker GJ, Alexander DA. Camino: Open-Source Diffusion-MRI Reconstruction and Processing. 2006; Seattle, WA, USA. p P2759.

20. Hall MG, Bongers A, Sved P, Watson G, Bourne RM. Assessment of non-Gaussian diffusion with singly and doubly stretched biexponential models of DWI signal attenuation in prostate tissue. NMR in Biomedicine 2015;28:486-495.

21. Bourne RM, Panagiotaki E, Bongers A, Sved P, Watson G, Alexander DC. Information theoretic ranking of four models of diffusion attenuation in fresh and fixed prostate tissue ex vivo. Magnetic Resonance in Medicine 2014;72:1418-1426.

22. Bourne R, Bongers A, Charles N, Power C, Sved P, Watson G. Effect of formalin fixation on biexponential modeling of diffusion decay in prostate tissue. Magnetic Resonance in Medicine 2013;70(4):1160-1166.

23. Bourne R, Kurniawan N, Cowin G, Chowdhury S, Sved P, Watson G, Stait-Gardner T, Price W. Biexponential Diffusion Decay in Formalin Fixed Prostate Tissue: Preliminary Findings. Magnetic Resonance in Medicine 2012;68(3):954-959.

24. Shinmoto H, Oshio K, Tanimoto A, Higuchi N, Okuda S, Kuribayashi S, Mulkern RV. Biexponential apparent diffusion coefficients in prostate cancer. Magnetic resonance imaging 2009;27(3):355-359.

25. Jonmarker S, Valdman A, Lindberg A, Hellstrom M, Egevad L. Tissue shrinkage after fixation with formalin injection of prostatectomy specimens. Virchows Archiv 2006;449(3):297-301.

26. Chatterjee A, McEntee M, Watson G, Myint E, Bourne RM. Changes in epithelium, stroma, and lumen space correlate more strongly with prostate cancer Gleason grade than cellularity metrics. Radiology 2015;277(3):751-762. 
Tables

Table 1. 6-direction DTI acquisition parameters

\begin{tabular}{|c|c|c|c|c|c|c|c|c|c|c|}
\hline Prostate & $\begin{array}{c}\text { FOV } \\
\text { (matrix) }\end{array}$ & $\begin{array}{c}\text { Voxel size } \\
(\mathrm{mm})\end{array}$ & $\begin{array}{c}\mathrm{TR} \\
\text { (ms) }\end{array}$ & $\begin{array}{l}b \text {-value }{ }^{a} \\
\left(\mathrm{~s} / \mathrm{mm}^{2}\right)\end{array}$ & $\begin{array}{l}\Delta^{\mathrm{b}} \\
(\mathrm{ms})\end{array}$ & $\begin{array}{c}\mathrm{TE} \\
(\mathrm{ms})\end{array}$ & AV & $\begin{array}{l}\text { SNR } \\
b=0\end{array}$ & $\begin{array}{c}\text { SNR } \\
b=800\end{array}$ & $\begin{array}{c}\text { SNR } \\
b=1600\end{array}$ \\
\hline \multirow{10}{*}{1} & \multirow{10}{*}{$\begin{array}{c}50 \times 50 \\
\mathrm{~mm} \\
(40 \times 40)\end{array}$} & \multirow{10}{*}{$1.25 \times 1.25 \times 2$} & \multirow{10}{*}{2200} & \multirow{10}{*}{$\begin{array}{c}800 \\
1600\end{array}$} & 10 & 18 & 2 & 190 & 95 & 62 \\
\hline & & & & & 20 & 28 & 2 & 137 & 74 & 50 \\
\hline & & & & & 30 & 38 & 2 & 119 & 66 & 45 \\
\hline & & & & & 40 & 48 & 2 & 105 & 59 & 40 \\
\hline & & & & & 50 & 58 & 2 & 55 & 31 & 21 \\
\hline & & & & & 60 & 68 & 4 & 89 & 50 & 35 \\
\hline & & & & & 70 & 78 & 4 & 47 & 27 & 19 \\
\hline & & & & & 80 & 88 & 8 & 57 & 32 & 22 \\
\hline & & & & & 90 & 98 & 8 & 50 & 28 & 19 \\
\hline & & & & & 100 & 108 & 8 & 30 & 16 & 12 \\
\hline \multirow{11}{*}{2} & \multirow{11}{*}{  } & \multirow{11}{*}{$1.25 \times 1.25 \times 2$} & \multirow{11}{*}{2200} & \multirow{11}{*}{$\begin{array}{c}800 \\
1600\end{array}$} & 10 & 18 & 2 & 311 & 153 & 97 \\
\hline & & & & & 20 & 28 & 2 & 211 & 113 & 75 \\
\hline & & & & & 30 & 38 & 2 & 173 & 95 & 64 \\
\hline & & & & & 40 & 48 & 2 & 103 & 57 & 39 \\
\hline & & & & & 50 & 58 & 2 & 78 & 44 & 30 \\
\hline & & & & & 60 & 68 & 4 & 105 & 59 & 41 \\
\hline & & & & & 70 & 78 & 4 & 75 & 42 & 29 \\
\hline & & & & & 80 & 88 & 4 & 63 & 35 & 25 \\
\hline & & & & & 90 & 98 & 4 & 38 & 21 & 15 \\
\hline & & & & & 100 & 108 & 4 & 28 & 16 & 11 \\
\hline & & & & & 120 & 128 & 4 & 18 & 10 & 7 \\
\hline \multirow{10}{*}{3} & \multirow{10}{*}{$\begin{array}{c}60 \times 60 \\
\mathrm{~mm}\end{array}$} & \multirow{10}{*}{$1.5 \times 1.5 \times 2$} & \multirow{10}{*}{2600} & \multirow{10}{*}{$\begin{array}{c}800 \\
1600\end{array}$} & 10 & 18 & 2 & 208 & 95 & 60 \\
\hline & & & & & 20 & 28 & 2 & 165 & 82 & 53 \\
\hline & & & & & 30 & 38 & 2 & 150 & 76 & 49 \\
\hline & & & & & 40 & 48 & 2 & 140 & 71 & 46 \\
\hline & & & & & 50 & 58 & 2 & 109 & 55 & 36 \\
\hline & & & & & 60 & 68 & 4 & 147 & 74 & 48 \\
\hline & & & & & 70 & 78 & 4 & 74 & 37 & 24 \\
\hline & & & & & 80 & 88 & 8 & 137 & 68 & 44 \\
\hline & & & & & 90 & 98 & 8 & 101 & 50 & 33 \\
\hline & & & & & 100 & 108 & 8 & 77 & 37 & 24 \\
\hline
\end{tabular}

a) Nominal $b$-value. Effective $b$-values were used for model fitting.

b) $\delta=5 \mathrm{~ms}$ for all measurements. 
Table 2.

3-direction DWI acquisition parameters

\begin{tabular}{|c|c|c|c|c|c|c|c|c|}
\hline Prostate & $\begin{array}{c}\text { FOV } \\
\text { (matrix) }\end{array}$ & $\begin{array}{l}\text { Voxel size } \\
(\mathrm{mm})\end{array}$ & $\begin{array}{l}\mathrm{TR} \\
(\mathrm{ms})\end{array}$ & $\begin{array}{l}b \text {-value }{ }^{a} \\
\left(\mathrm{~s} / \mathrm{mm}^{2}\right)\end{array}$ & $\begin{array}{l}\Delta^{\mathrm{b}} \\
(\mathrm{ms})\end{array}$ & $\begin{array}{c}\mathrm{TE} \\
(\mathrm{ms})\end{array}$ & $\mathrm{AV}$ & $\begin{array}{l}\text { SNR } \\
b=0\end{array}$ \\
\hline \multirow{4}{*}{1} & \multirow{4}{*}{$\begin{array}{c}50 \times 50 \mathrm{~mm} \\
(40 \times 40)\end{array}$} & \multirow{4}{*}{$1.25 \times 1.25 \times 2$} & \multirow{4}{*}{2000} & \multirow{4}{*}{$\begin{array}{c}100,311,603 \\
965,1391 \\
1873,2411 \\
3000\end{array}$} & 10 & 18 & 1 & 133 \\
\hline & & & & & 20 & 28 & 1 & 123 \\
\hline & & & & & 40 & 48 & 1 & 83 \\
\hline & & & & & 80 & 88 & 2 & 29 \\
\hline \multirow{4}{*}{2} & \multirow{4}{*}{$\begin{array}{c}50 \times 50 \mathrm{~mm} \\
(64 \times 64)\end{array}$} & \multirow{4}{*}{$0.78 \times 0.78 \times 2$} & \multirow{4}{*}{2200} & \multirow{4}{*}{$\begin{array}{c}100,311,603 \\
965,1391 \\
1873,2411 \\
3000\end{array}$} & 10 & 18 & 4 & 448 \\
\hline & & & & & 20 & 28 & 4 & 270 \\
\hline & & & & & 40 & 48 & 4 & 164 \\
\hline & & & & & 80 & 88 & 4 & 56 \\
\hline \multirow{4}{*}{3} & \multirow{4}{*}{$\begin{array}{c}60 \times 60 \mathrm{~mm} \\
(40 \times 40)\end{array}$} & \multirow{4}{*}{$1.5 \times 1.5 \times 2$} & \multirow{4}{*}{2600} & \multirow{4}{*}{$\begin{array}{c}100,311,603 \\
965,1391 \\
1873,2411 \\
3000\end{array}$} & 10 & 18 & 1 & 152 \\
\hline & & & & & 20 & 28 & 1 & 134 \\
\hline & & & & & 40 & 48 & 1 & 90 \\
\hline & & & & & 80 & 88 & 1 & 44 \\
\hline
\end{tabular}

a) Nominal $b$-value. Effective $b$-values were used for model fitting.

b) $\delta=5 \mathrm{~ms}$ for all measurements.

Table 3.

Zeppelin-sphere model parameters

\begin{tabular}{|c|c|c|}
\hline Parameter & Description & Fitting constraints \\
\hline Zeppelin $f$ & $\begin{array}{c}\text { Fraction of signal due to zeppelin } \\
\text { component }\end{array}$ & $0-1$ \\
\hline Zeppelin $D_{\text {II }}$ & $\begin{array}{c}\text { Diffusivity parallel to the zeppelin } \\
\text { long axis }\end{array}$ & $0-2.1 \mu \mathrm{m}^{2} / \mathrm{ms}$ \\
\hline Zeppelin $D_{\perp}$ & $\begin{array}{c}\text { Diffusivity perpendicular to the } \\
\text { zeppelin long axis }\end{array}$ & $0-2.1 \mu \mathrm{m}^{2} / \mathrm{ms}$ \\
\hline Sphere $D$ & Diffusivity inside the sphere & $0-20 \mu \mathrm{m}$ \\
\hline Sphere $R$ & Sphere radius & $0-2.00$ \\
\hline
\end{tabular}




\section{Figure Captions}

Figure 1. Calculation of ADC and FA from DTI and DWI data using the open source CAMINO toolkit.

To maximize the independence of the measured and predicted ADC and FA values the zeppelinsphere model fit used just one of the multi- $\Delta$ DTI measurements to define the orientation of the zeppelin component.

Figure 2. Effect of diffusion time on mean voxel ADC and FA.

Data represent the average of 4837, 4838, and 6016 intraprostatic voxels in Prostates 1, 2, and 3 respectively. Note that the SNR plots are based on the signal measured at $b=800$ and 1600 $\mathrm{s} / \mathrm{mm}^{2}$, rather than the more conventional but less informative ' $b=0$ ' signal.

Fig. 3. Parameter maps for Zeppelin-sphere model.

Maps show a single mid-organ slice for each prostate.

Figure 4. Measured ADC and FA versus ADC and FA predicted by the 'Zeppelin-sphere' model.

2D-histogram contours based on voxel count for $b=800 \mathrm{~s} / \mathrm{mm}^{2}$ data. Very similar plots were obtained for $b=1600 \mathrm{~s} / \mathrm{mm}^{2}$ (data not shown).

Figure 5. Correlation of measured FA and Zeppelin FA.

2D histogram contour plot based on voxel count. Data for $b=800 \mathrm{~s} / \mathrm{mm}^{2}$.

Supporting Figure S1. Examples of high quality of the fit of the 'zeppelin-sphere' model.

Plots show the fit to 3-direction multi-b data combined with $\Delta=20 \mathrm{~ms}$ DTI data. Representative selection of voxels from three prostates. 


\section{Figures}

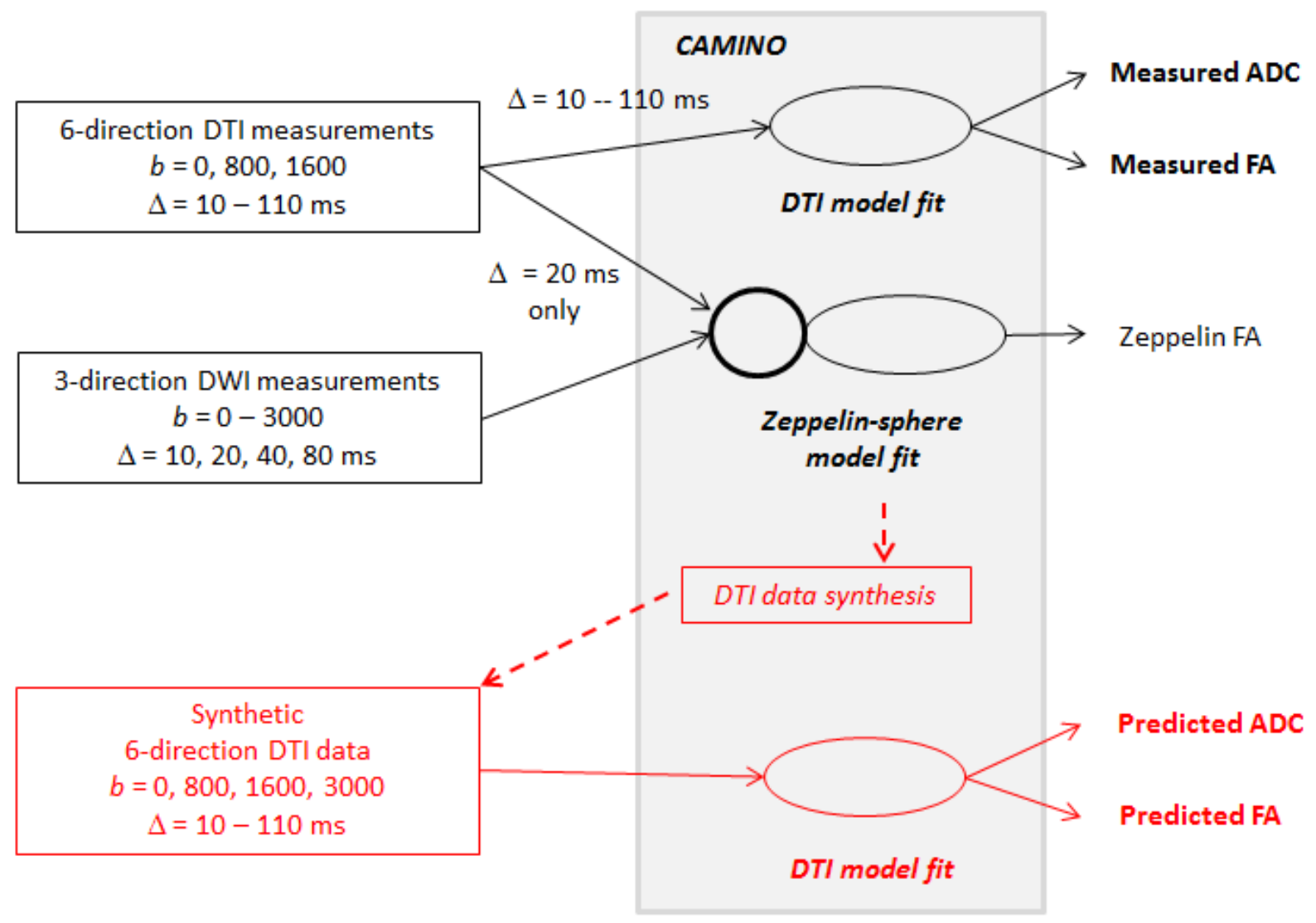

Figure 1. Calculation of ADC and FA from DTI and DWI data using the open source CAMINO toolkit.

To maximize the independence of the measured and predicted ADC and FA values the zeppelinsphere model fit used just one of the multi- $\Delta$ DTI measurements to define the orientation of the zeppelin component. 

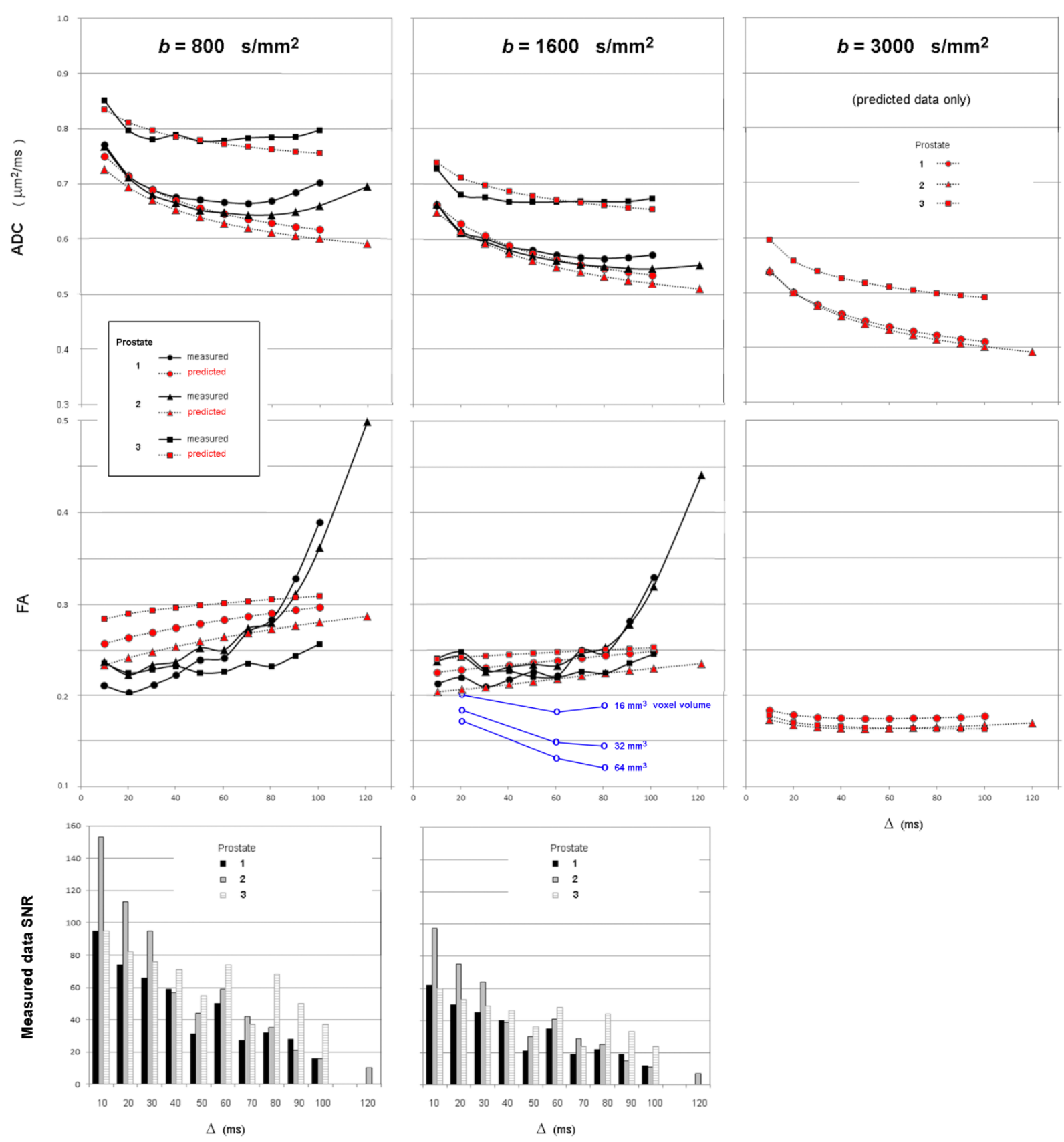

Figure 2. Effect of diffusion time on mean voxel ADC and FA.

Data represent the average of 4837, 4838, and 6016 intraprostatic voxels in Prostates 1, 2, and 3 respectively. Note that the SNR plots are based on the signal measured at $b=800$ and 1600 $\mathrm{s} / \mathrm{mm}^{2}$, rather than the more conventional but less informative ' $b=0$ ' signal. For reference, data 
from previously reported measurements at larger voxel sizes (Bourne et al. DOI $10.1002 / \mathrm{mrm} .25908$ ) are shown in blue. 


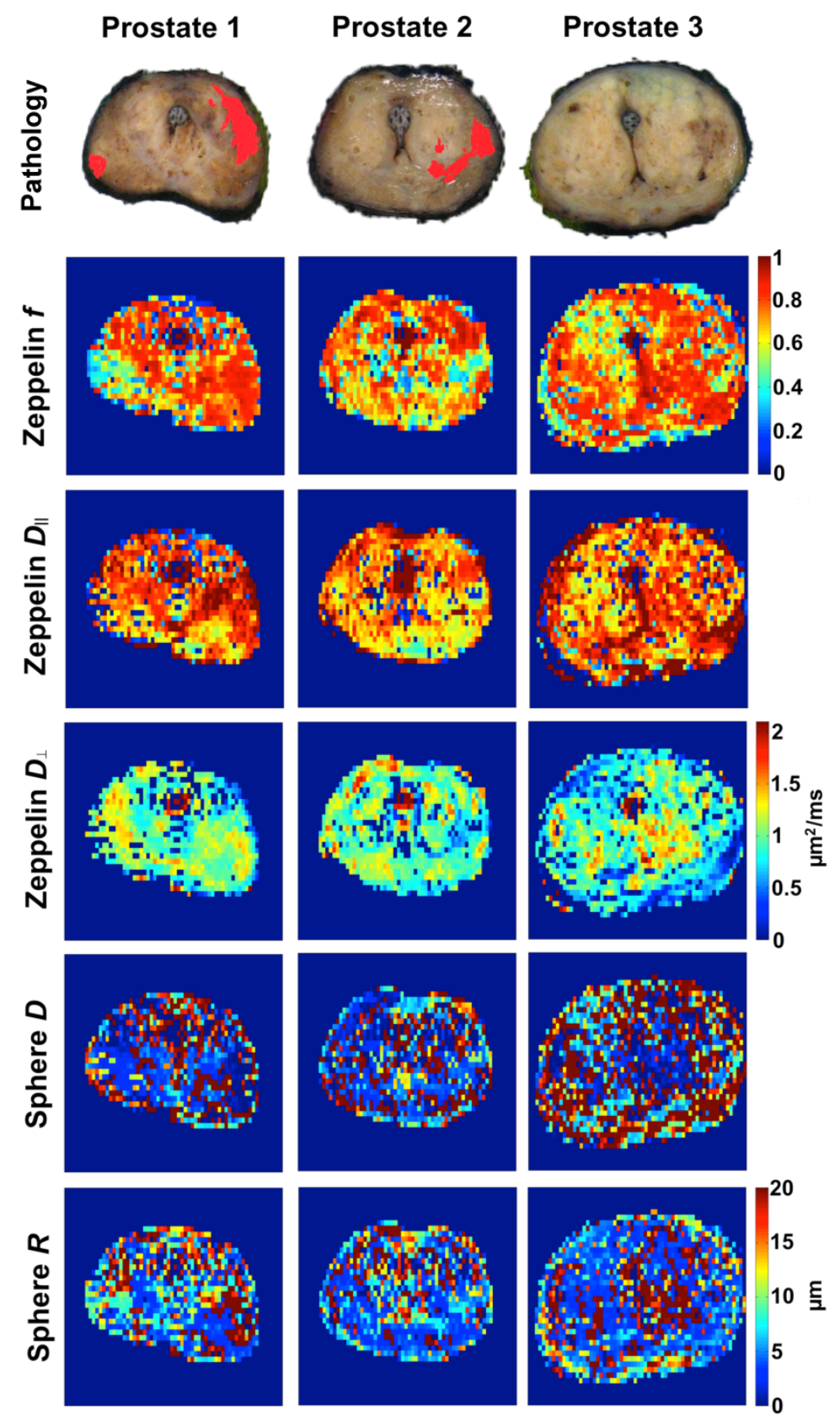

Fig. 3. Parameter maps for Zeppelin-sphere model.

Maps show a single mid-organ slice for each prostate. Approximately aligned pathology maps are shown for reference, with Gleason pattern 4 cancer coloured red. 

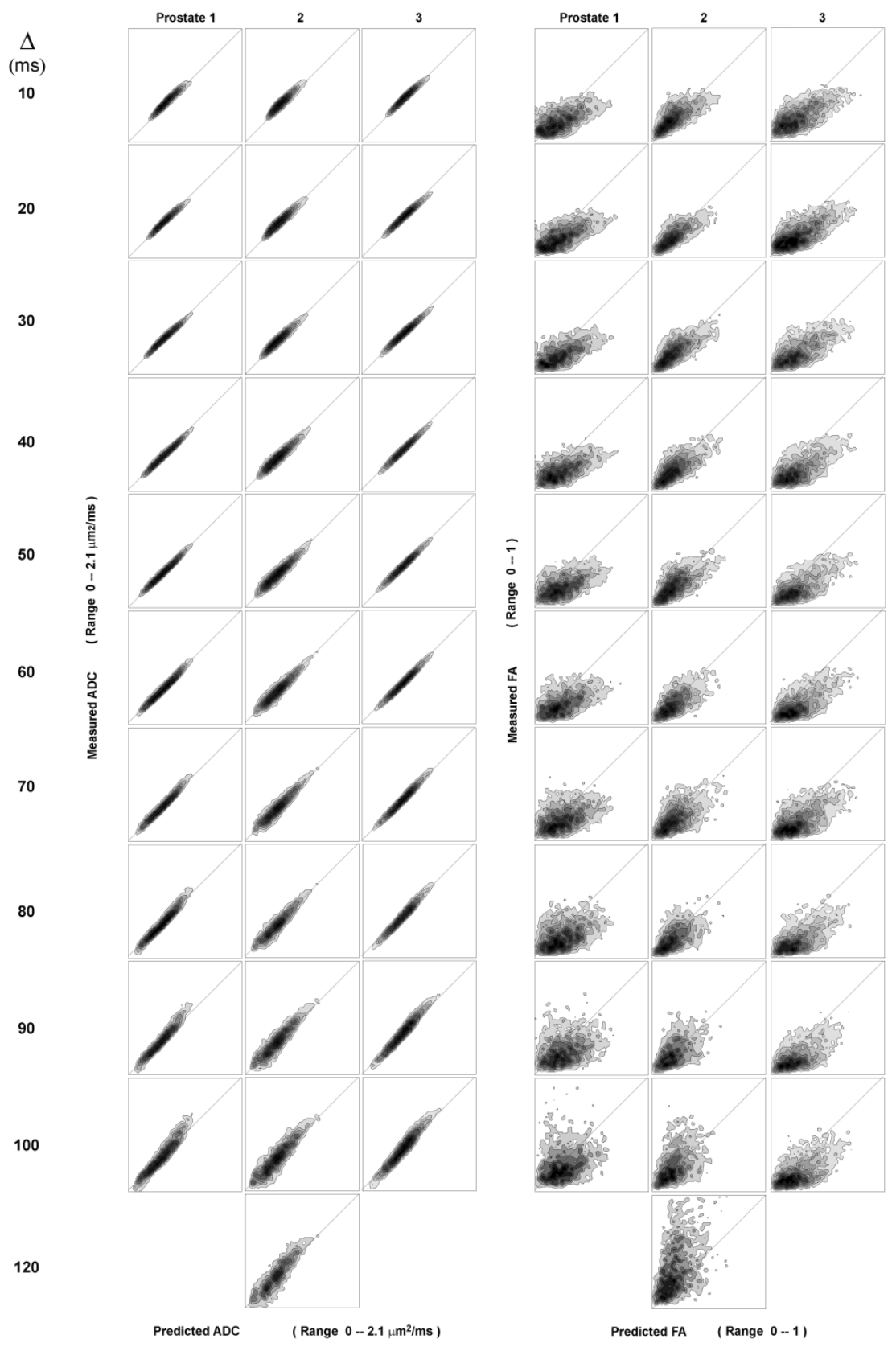

Figure 4. Measured ADC and FA versus ADC and FA predicted by the 'Zeppelin-sphere' model.

2D-histogram contours based on voxel count for $b=800 \mathrm{~s} / \mathrm{mm}^{2}$ data. Very similar plots were obtained for $b=1600 \mathrm{~s} / \mathrm{mm}^{2}$ (data not shown). 


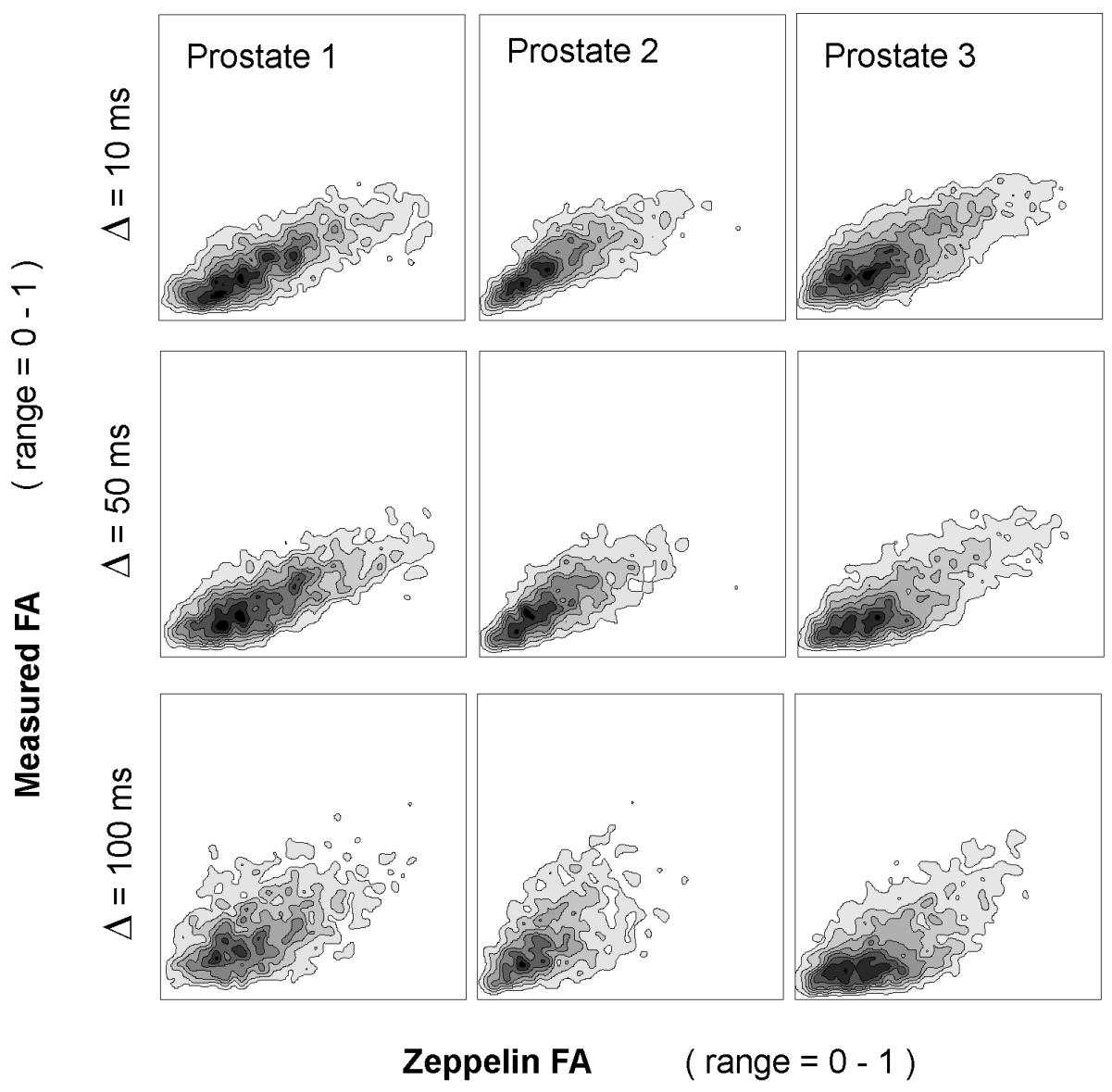

Figure 5. Correlation of measured FA and Zeppelin FA.

2D histogram contour plot based on voxel count. Data for $b=800 \mathrm{~s} / \mathrm{mm}^{2}$. 